Ann. Génét. Sél. anim., I974, 6 (4), 399-404.

\title{
PIGMENTS MÉLANIQUES DU PELAGE DE QUELQUES RACES BOVINES : LE SCHÉMA POSSIBLE DE CERTAINES ACTIONS GÉNIQUES
}

\author{
Giovanna MISURACA, G. PROTA et J.-J. LAUVERGNE* \\ Istituto di Chimica organica e biologica, \\ Università di Napoli, \\ Via Mezzocannone 16, Napoli \\ * Département de Génétique animale, \\ Centre national de Recherches zootechniques, I. N.R. A., \\ 78350 Jouy en Josas
}

\begin{abstract}
RÉSUMÉ
A l'instar des autres espèces de Mammifères et d'Oiseaux déjà étudiées, certaines colorations observées dans des races bovines rouges sont dues aux phaeomélanine. Ces pigments exigent pour leur synthèse, outre la présence de tyrosine oxydée, celle de cystéine. Ils diffèrent définitivement des eumélanines, les pigments noirs présents dans des races bovines noire et gris souris. Comme une action enzymatique est nécessaire pour la production des phaeomélanines, on propose d'attribuer la fabrication de cette enzyme au locus agouti (A) dont la présence constante dans beaucoup d'espèces de Mammifères est attestée par des séries alléliques phénotypiquement homologues et où il contrôle le dosage en eu- et phaeomélanine dans le poil et dans le pelage. Ce locus serait apparu dans le phyllum Mammifères-Oiseaux avant le clivage de ces deux ordres car les oiseaux ont aussi de la phaeomélanine. Dans les ordres de Vertébrés inférieurs, en effet, on constate la présence des seules eumélanines. Le locus A semble présent chez les Bovins, d'après la série phénotypiquement parfaitement homologable à celle des Mammifères mieux étudiés. Toutefois, dans cette espèce, comme d'ailleurs chez d'autres ruminants, dans des cas où ils devraient apparaître en alternance avec les eumélanines les pigments phaeomélaniques ne se manifestent pas, en particulier dans le patron coloré dit provisoirement "agouti à ventre clair " de la Brune des Alpes dont la couleur est gris-souris.
\end{abstract}

Jusqu'à une époque récente les pigments rouges appelés phaeomélanines, parfois présents dans les phanères des Oiseaux et des Mammifères, étaient considérés comme chimiquement très proches des eumélanines ou mélanines noires, pigments azotés très courants dans le règne animal. Cependant, de récentes études (ProTA et NicoLAUS, I967 ; MisUraca et al., I970 ; ProTA, I972) ont montré que les phaeomélanines contenaient du soufre et résultaient d'une combinaison de la cystéine avec la dopa- 
quinone produite par oxydation enzymatique de la tyrosine et intermédiaire dans la formation des eumélanines (fig. I).

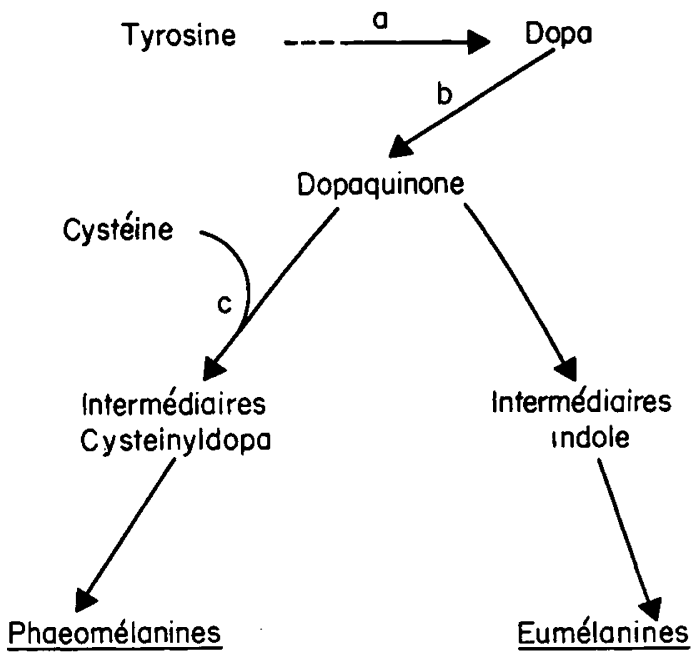

FIG. I. - Interrelations biogénétiques entre les eumélanines et les phaeomélanines Les réactions $a$ et $b$ sont contrôlées par la tyrosinase, $c$ n'est certainement pas enzymatique mais le contenu en cystéine du mélanocyte est probablement sous contrôle enzymatique

Ces considérations nouvelles ont amené à réexaminer la situation des pigments mélaniques dans les phanères des Mammifères et des Oiseaux. Une première série d'espèces comprenant la Poule, le Faisan, la Perdrix, le Pigeon, le Lapin, la Chèvre, le Mouton, le Sanglier, le Daim rouge et 1'Homme a été analysée (FATTorusso et al., I970). Dans le présent article, nous voudrions étendre cette étude aux Bovins domestiques. Il s'agit de préciser quels types de pigments sont présents dans les poils des phénotypes colorés les plus courants et de tenter de préciser l'action biochimique de gènes qui, jusqu'à présent, ne sont connus que par les actions visibles de leurs différents mutants.

\section{MATÉRIEL ET MÉTHODES}

$$
\text { I. - Matériel }
$$

On a prélevé du poil de races bovines noires (Frisonne Pie Noire), rouges (Valdotaine, Reggiana et Tachetée Rouge du Simmenthal) et gris-souris (Brune des Alpes).

En plus divers échantillons en provenance d'animaux croisés ont pu être examinés : Brune des Alpes $\times$ Piémontaise (gris-souris $\times$ gris) Brune des Alpes $\times$ Frisonne Pie Noive (gris-souris $\times$ noir).

\section{Eumélaines.}

$$
\text { 2. -- Méthodes }
$$

Il est facile de faire la différence entre ces pigments et les phaeomélanines, du fait de leur couleur plus sombre et, surtout, de leur insolubilité dans les alcalis dilués.

\section{Phaeomélanines.}

Les phaeomélanines se divisent en deux groupes : les trichochromes et les gallophaeomélanines. Les gallophaeomélanines ont un poids moléculaire élevé et sont difficiles à étudier. Par 
contre les trichochromes $\left(^{1}\right)$ constituent un groupe plus simple, de caractère amphotérique, avec un spectre d'absorption en UV caractéristique. En outre, ils peuvent être aisément identifiés par chromatographie sur papier.

Les gallophaeomélanines et les trichochromes vont toujours ensemble, la présence des seuls trichochromes est donc une preuve décisive que le pigment analysé appartient au groupe des phaeomélanines. Comme, par ailleurs, la quantité de matériel disponible était réduite on s'est limité à l'étude des trichochromes en utilisant la méthode suivante :

Un échantillon de 20 à $30 \mathrm{~g}$ de poils est mis dans du chloroforme, laissé à la température ambiante pendant une nuit, puis lavé deux fois dans l'acétone et plusieurs fois dans l'éthanol bouillant. Ensuite, on procède à l'extraction avec de la soude à $0, \mathrm{I} N$, à la température ambiante. Après 6 heures, l'extrait est décanté et le résidu réextrait plusieurs fois, jusqu'à ce que le surnageant soit presque incolore. Après filtration les extraits mélangés sont ramenés à pH I avec $\mathrm{HCl} 6 \mathrm{~N}$ chauffé à $80^{\circ}$ pendant 30 minutes. A cette étape de l'analyse les trichochromes $\mathrm{B}$ et $\mathrm{C}$ subissent une décarboxylation donnant les dérivés décarboxylés correspondants. Ensuite on centrifuge pour enlever les pigments insolubles dans l'acide et/ou les protéines. Le surnageant est alors passé dans une colonne $(\mathrm{I} \times 5 \mathrm{~cm})$ de Dowex $50 \mathrm{~W}-\mathrm{X}_{2}$ (100-200 mesh, forme $\mathrm{H}^{+}$); après lavage avec $\mathrm{HCl}$ IN et eau distillée les biochromes sont séparés de la résine par $\mathrm{NaOH} 0,5 \mathrm{~N}$. L'éluat est acidifié à $\mathrm{pH}$ I avec $\mathrm{HCl} 6 \mathrm{~N}$ et analysé par chromatographie sur papier Whatman $\mathrm{n}^{\circ} \mathrm{I}$ (en technique descendante) en utilisant comme éluant un mélange de n-butanol, d'acide acétique et d' $\mathrm{HCl} 0,3 \mathrm{~N}(20: 30: 50 \mathrm{v} / \mathrm{v})$.

\section{RÉSULTATS}

Lorsqu'ils étaient traités avec $\mathrm{NAOH}$ o,IN à la température ambiante, les poils de certaines races donnaient des extraits incolores dans lesquels il n'était pas possible d'extraire de phaeomélanines, ce qui indiquait que seul les pigments eumélaniques étaient présents. Les autres échantillons, au contraire, ne présentaient que des pigments de type phaeomélanique. La situation est résumée dans le tableau I.

TABLEAU I

Mise en évidence des deux types de mélanine dans les poils de quelques races bovines

\begin{tabular}{|c|c|c|c|}
\hline & \multirow{2}{*}{$\begin{array}{l}\text { Couleur } \\
\text { du poil }\end{array}$} & \multicolumn{2}{|c|}{$\begin{array}{c}\text { Types de pigments } \\
\text { décelés (*) }\end{array}$} \\
\hline & & $\begin{array}{l}\text { phoeomé- } \\
\text { laniques }\end{array}$ & $\begin{array}{l}\text { eumé- } \\
\text { laniques }\end{array}$ \\
\hline $\begin{array}{l}\text { Frisonne Pie Noire } \\
\text { Valdotaine } \\
\text { Reggiana } \\
\text { Tachetée rouge Simmental } \\
\text { Brune des Alpes } \\
\text { Brune des Alpes } \times \text { Piémontaise } \\
\text { Frisonne } \times \text { Brune des Alpes }\end{array}$ & $\begin{array}{l}\text { noire } \\
\text { rouge } \\
\text { rouge } \\
\text { rouge } \\
\text { gris-souris } \\
\text { gris-souris } \\
\text { noire }\end{array}$ & $\begin{array}{l}- \\
+ \\
+ \\
+ \\
- \\
-\end{array}$ & $\begin{array}{l}+ \\
- \\
- \\
+ \\
+ \\
+\end{array}$ \\
\hline $\begin{array}{c}\left(^{*}\right)+\text { présence } \\
\text { - absence }\end{array}$ & & & \\
\hline
\end{tabular}

(1) Dans le présent article on emploiera le terme plus approprié de trichochrome à la place du terme trichosidérine précédemment employé. Il a été en effet, récemment démontré que cette substance ne contenait pas de fer (THOMSON, 1974). 
L'analyse des échantillons contenant de la phaeomélanine s'est alors déroulée selon les méthodes décrites ci-dessus et a donné les résultats consignés dans le tableau 2.

TABLEAU 2

Caractéristiques des pigments phoeomélaniques trichochromes des divers échantillons qui en présentaient

\begin{tabular}{|c|c|c|c|c|}
\hline \multirow{2}{*}{ Espèces et races } & \multicolumn{4}{|c|}{ Trichochromes } \\
\hline & $\mathrm{B}\left({ }^{(1)}\right.$ & $C\left({ }^{1}\right)$ & $\mathbf{E}$ & $\mathrm{F}$ \\
\hline \multicolumn{5}{|l|}{ Bovins } \\
\hline $\begin{array}{l}\text { Valdotaine } \\
\text { Reggiana } \\
\text { Simmental }\end{array}$ & $\begin{array}{l}++ \\
++ \\
+t\end{array}$ & $\begin{array}{l}+t+t \\
+++t \\
++t+\end{array}$ & $\begin{array}{c}0 \\
+ \\
\text { traces }\end{array}$ & $\begin{array}{c}0 \\
+ \\
\text { traces }\end{array}$ \\
\hline
\end{tabular}

(1) Les trichochromes $\mathrm{B}$ et $\mathrm{C}$ sont instables en milieu acide et, au cours de l'extraction ils subissent une décarboxylation en $\mathrm{C}-3$ pour donner les dérivés décarboxylés correspondants.

\section{DISCUSSION}

Le tableau I montre d'abord, qu'à l'instar des autres espèces déjà étudiées de Mammifères et d'Oiseaux (FATrorusso et al., I970), les poils des bovins peuvent présenter les deux types de pigments mélaniques. Les races noires (Frisonne) portent de l'eumélanine, les races rouges (Valdotaine, Reggiana) de la phaéomélanine. Ce résultat n'est pas aussi évident qu'on pourrait le croire à première vue car, si la phaeomélanine paraît toujours rouge, il n'en est pas de même de l'eumélanine souvent sombre mais qui, sous diverses influences, peut prendre des nuances variées. Par exemple, il existe dans plusieurs espèces un mutant au locus appelé $B$ qui peut la rendre brune (SEARLE, I968) mais la couleur brune peut aussi résulter d'une oxydation du pigment noir. Quand le poil a une seule couleur, dans les bruns-rouges, une telle étude biochimique est donc nécessaire.

Le tableau 2 montre que, dans les races rouges (qui présentent seulement des phaeomélanines) il y a une certaine variation de la teneur en trichochromes $\mathrm{E}$ et $\mathrm{F}$. Il pourrait être intéressant de relier cette variabilité avec des nuances de la couleur du pelage ou avec certaines actions géniques.

Chez les Mammifères, à la suite d'études comparées des séries alléliques faites principalement chez les Rongeurs et les Carnivores (HALDANE, I927 ; LITTTLE, I958) on reconnaît comme très constante la présence d'un locus dit Agouti (A) qui contrôle la répartition des eu- et des phaeomélanines à travers le poil (donnant parfois des poils zonés) et à travers le manteau (donnant un certain nombre de "patrons "symétriques 
(comme agouti, agouti ventre clair, tout noir, noir et feu...) (cf. SEARLE, I968, pour revue plus récente).

Cherchant une correspondance gène/enzyme dans le système qui produit les pigments mélaniques on doit d'abord se rappeler qu'une action enzymatique est nécessaire et suffisante pour la production d'eumélanine : celle de la tyrosinase. Certains auteurs comme FOSTER (I966) sont alors enclins à penser que le gène de structure qui contrôle cet enzyme serait, chez les Mammifères, précisément le gène $\mathrm{C}$ dit d'albinisme, dont une série allélique quasi stéréotypée a été mis en évidence dans plusieurs espèces.

Pour la formation des phaeomélanines les études de Nicolaus (I972) ont montré 1a nécessité d'une autre action enzymatique qui consisterait à régler le contenu en cystéine des mélanocytes. Il est alors tentant d'attribuer ce rôle de régulation de la cystéine dans le mélanocyte au locus Agouti évoqué plus haut. Ses manifestations visibles consistent précisément à doser le mélange d'eu- et de phaeomélanines dans le poil et, plus généralement, dans tout le manteau. Chez les Bovins, si les tests d'allélisme n'ont pu être menés aussi loin que dans les espèces de Rongeurs et de Carnivores passées en revue par SEARLE (I968) on peut néanmoins identifier une série de " patrons " colorés parfaitement homologables phénotypiquement aux séries $\mathrm{A}$ des espèces où l'expérimentation mendélienne est plus facile (LAUVERGNE, r966). Cette homologation phénotypique pourrait même - avec la découverte récente d'un phénotype noir et feu (LAUVERGNE, I970) et avec la prise en compte des termes rouge et noir dont l'alternance avait été, probablement à tort, laissée au jeu d'allèles à un autre locus : le locus E, dit d'extension - comprendre au moins 6 termes qui seraient, provisoirement, les suivants $\left(^{1}\right)$ :

As (s pour solid) : Non agouti (tout noir), ex : race Angus

$\mathrm{A}^{+}$Agouti (dun), ex : dun du Galloway

$\mathrm{A}^{y}$ ( $y$ pour yellow) : Jaune à extrémités noires : ex : race $A u b r a c$

$\mathrm{A}^{w}$ (w pour white belly) : Agouti ventre clair, ex : Race Brune des Alpes

$\mathrm{A}^{t}$ ( $t$ pour black and tan) : Noir et feu, ex : certains zébus de Madagascar

$\mathrm{A}^{r} \quad(r$ pour red) : Rouge, ex : Simmental

Il est à remarquer que, dans les patrons présentant des poils zonés comme celui de la Brune des Alpes, que nous avons attribué à l'allèle Aw (Agouti ventre blanc) et où l'alternance des bandes claires et foncées a été remarquée pour la première fois par BERGE, I96I, la phaeomélanine n'existe pas (cf. tab1. I). Ce comportement de la phaeomélanine, qui disparaît de certains patrons colorés où l'alternance est de règle chez les Rongeurs et les Carnivores, ne semble pas rare chez d'autres Ruminants, comme nous l'avons noté précédemment (LAUVERGNE, I970) chez la Chèvre et le Mouton).

On peut enfin remarquer que les phaeomélanines sont absentes chez les Poissons et les Reptiles et penser que ce gène Agouti, qui contrôlerait l'introduction de la cystéine dans les mélanocytes a pu faire son apparition dans le phyllum qui a conduit aux Oiseaux et aux Mammifères, avant sa différenciation.

Reçu pour publication en décembre 1974.

(1) A la lecture du manuscrit, le Dr SEArLe a fait remarquer qu'il sera souhaitable d'adopter une nomenclature allélique calquée sur celle de la Souris, c'est-à-dire en désignant par des lettres minuscules les allèles récessifs à l'allèle sauvage. Cela n'est malheureusement guère possible pour les bovins domestiques: tout d'abord notre choix de l'allèle sauvage (exposant +) est très arbitraire, en outre l'ordre de dominance n'est encore connu que très approximativement. 


\section{REMERCIEMENTS}

Les échantillons de poils ont été fournis par W. STuder Institut für Zuchthygiene Universitäts Zürich et par le Pr. Succi, Istituto de Zootecnia generale, Facolta di Agraria, Milano.

Le manuscrit a été lu par le Dr A. G. Searle Medical Research Council, Radiobiology Únit, Harwell, Didcot, Grande-Bretagne.

\section{SUMMARY}

\section{MELANIC PIGMENTS OF HAIR IN SOME CAT'TLE BREEDS : A POSSIBLE PLAN OF ACTION FOR CERTAIN GENES}

As other species of mammals and birds already studied, some red cattle breeds have their colorings due to phaeomelanins. These pigments require cysteine as well as oxydized tyrosine for synthesis. They are definitely different than the black pigments, eumelanines, whose existence has been prooved in black and in grey cattle. As enzyme action is necessary for phaeomelaninș production, we propose that production of this enzyme may be attributed to the agouti locus (A). The constant presence of this locus is attested to in many mammals by homologically phenotypic allelic series; it controls eumelanins and phaeomelanins determination in hair and fur.

This locus must have appeared in the mammal - bird phylum before the branching of these two orders because birds present also phaeomelanin. In fact, only eumelanins are present in the lower vertebrates. Locus A seems present in cattle according to the series, which is perfectly phenotypically homologable to that of better studied mammals. However, in that species, as in some other ruminants, phaeomelanic pigments are not present in some cases where they should appear alternatively with eumelanins, particularly in the color pattern provisionally called " white-belly agouti" in the Brown Alpine.

\section{RÉFÉRENCES BIBLIOGRAPHIQUES}

BERGE S., 1961. Influence of dun on brown and brindle in cattle. Z. Tierz. ZiuchtBiol., 75, 298-306.

Fattorusso E., Minale L., G. Sodano, 1970. Feomelanine e eumelanine da nuove fonti naturali. Gaz. chimica ital., 100, 452-460.

Foster M., 1967. Genetic aspects of mammalian melanogenesis, in Montagu W., Funan H. U., $A d v$. Biol. Skin., 8. The pigmentary Systems, 467-477, Pergamon Press, London.

Haldane J. B. S., r927. The comparative genetics of Colour in Rodents and Carnivora. Biol. Rev., 2, I99-2I2.

LAUvergne J. J., r966. Génétique de la couleur du pelage des Bovins domestiques (Bostaurus L.). Bibliogr. Genet., 20, I-68.

Lauvergne J.-J., r97o. Mise en évidence du phénotype noir et feu dans deux nouvelles espèces de Mammifères. Rev. Roum. Biol., sér. Zool., 15, II3-II8.

Litrle C. C., I958. Coat Color genes in Rodents and Carnivores. Quarterl. Rev. Biol., 33, ro3-I37.

Misuraca G., Nicolaus R. A. G., Prota, Ghiara G., r97o. A cytochemical Study of Phaeomelanin Formation in Feather Papillae of New Hampshire Chick embryos. Experientia, 25, 920-922.

Nicolaus R. A., I972. The nature of mammalian colors. Chimica Industria., 54, 427-433.

Prota G., Nicolaus R. A., r967. On the biogenesis of Phaeomelanins. Adv. Biol. Skin., 8. The pigmentary Systems, 323-328, Pergamon Press, London.

Prota G., 1972. Structure and Biogenesis of Phoeomelanins, in V. RILEY, Pigmentation : its genesis and biologic control, Appleton Century Croft, New York, 6r5-629.

Searle A. G., I968. Comparative Genetics of Coat Colour in Mammals. Logos Press, Academic Press. London.

Tномson R. N., I974. Pigment of reddish hairs and feathers. Angew. Chem. (Int. Edit.), 13, 305-312. 